EPJ manuscript No.

(will be inserted by the editor)

\title{
Nematic liquid crystals at rough and fluctuating interfaces
}

\author{
Jens Elgeti ${ }^{1 \mathrm{a}}$ and Friederike Schmid ${ }^{1}$ \\ Theoretische Physik, Universität Bielefeld, D-33501 Bielefeld, Germany \\ Received: date / Revised version: date
}

\begin{abstract}
Nematic liquid crystals at rough and fluctuating interfaces are analyzed within the Frank elastic theory and the Landau-de Gennes theory. We study specifically interfaces that locally favor planar anchoring. In the first part we reconsider the phenomenon of Berreman anchoring on fixed rough surfaces, and derive new simple expressions for the corresponding azimuthal anchoring energy. Surprisingly, we find that for strongly aligning surfaces, it depends only on the geometrical surface anisotropy and the bulk elastic constants, and not on the precise values of the chemical surface parameters. In the second part, we calculate the capillary waves at nematic-isotropic interfaces. If one neglects elastic interactions, the capillary wave spectrum is characterized by an anisotropic interfacial tension. With elastic interactions, the interfacial tension, i.e., the coefficient of the leading $q^{2}$ term of the capillary wave spectrum, becomes isotropic. However, the elastic interactions introduce a strongly anisotropic cubic $q^{3}$ term. The amplitudes of capillary waves are largest in the direction perpendicular to the director. These results are in agreement with previous molecular dynamics simulations.
\end{abstract}

PACS. $61.30 \mathrm{Hn}$ Liquid crystals: Surface phenomena - 61.30 Dk Continuum theories of liquid crystal structure - 68.05 -n Liquid-liquid interfaces

\section{Introduction}

Nematic liquid crystals are fluids with long range orientational order [1]. Compared to interfaces and surfaces in simple fluids, surfaces of nematic fluids have several peculiarities. First, the interface orients the nematic fluid [2, 3.4. This phenomenon, called surface anchoring, is quite remarkable, because it implies that the surface has direct influence on a bulk property of the adjacent fluid. It also has well-known practical applications in the LCD (liquid crystal display) technology [5]. Surface anchoring is driven by energetic and geometric factors, and depends on the structure of the surface. Second, the oriented nematic fluid breaks the planar symmetry of the interface. This should influence the properties of free interfaces, e.g., the spectrum of capillary wave fluctuations. Third, the nematic fluid is elastic in a generalized sense, i.e., fluctuations of the local orientation (the director) have long range correlations 6]. Since interfacial undulations and director fluctuations are coupled by means of the surface anchoring, this should introduce long range elastic interactions between the undulations. Hence interesting effects can be expected from the interplay of surface undulations and director fluctuations in liquid crystals [7].

In the present paper, we examine these phenomena in the framework of two continuum theories - the Frank elastic theory and the Landau-de Gennes theory. To separate

\footnotetext{
a Present address: Institute of Solid State Research, Research Center Jülich, D-52425 Jülich, Germany
}

the different aspects of the problem, we first consider a nematic liquid crystal in contact with a fixed, rough or patterned surface (Sec. 2). In LCDs, alignment layers are often prepared by coating them with polymers (polyimides) and then softly brushing them in the desired direction of alignment $5,8,9$. Assuming that brushing creates grooves in the surface 10, the success of the procedure indicates that liquid crystals tend to align in the direction where the surface modulations are smallest. Similarly, liquid crystals exposed to surfaces with stripelike gratings were found to align parallel to the stripes 11,12,13 14. Molecular factors of course contribute to this phenomenon, but the effect can already be explained on the level of the elastic theory. This was first shown by Berreman [15, and the theory was later refined by different authors [16 17. 18. Here we reconsider the phenomenon and derive simple new expressions for the anchoring angle and the anchoring strength.

In the second part (Sec. 3), we consider the capillary wave spectrum of free nematic/isotropic interfaces. Capillary waves are soft mode fluctuations of fluid-fluid interfaces, that are present even in situations where fluctuations can otherwise bee neglected. They were first predicted by Smoluchowski 19, and the theory was later worked out by various authors $[20,21,22,23,24,25,26]$. Since then they were observed in various systems experimentally $\left[\begin{array}{ll|l|l|l|l|}27 & 28,29 & 30 & 31\end{array}\right.$ as well as in computer simulations 32 ,

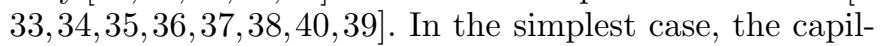
lary wave spectrum is governed by the free energy cost 
Jens Elgeti, Friederike Schmid: Nematic liquid crystals at rough and fluctuating interfaces

of the interfacial area that is added due to the undulations. Assuming that the fluctuating interface position can be parametrized by a single-valued function $h(x, y)$, and that local distortions $\partial h / \partial x$ and $\partial h / \partial y$ are small, the thermally averaged squared amplitude of fluctuations with wavevector $\mathbf{q}$ is predicted to be

$$
\left\langle|h(\mathbf{q})|^{2}\right\rangle=\frac{k_{B} T}{\sigma q^{2}}
$$

where $\sigma$ is the interfacial tension. Note that $\left\langle|h(\mathbf{q})|^{2}\right\rangle$ diverges in the limit $q \rightarrow 0$, hence the capillary waves with long wavelengths are predicted to be quite large. In real systems, however, the two coexisting fluids usually have different mass densities, and the gravitation introduces a low-wavelength cutoff in Eq. (1).

In the last years, capillary waves have attracted renewed interest in the context of soft condensed matter science. This is mainly due to the fact that typical interfacial tensions in soft materials are low, typical length scales are large, and coexisting phases often have very similar mass densities. Therefore, the capillary wave amplitudes in soft materials tend to be much larger than in simple fluids. For example, capillary waves were shown to have a significant effect on experimentally measured interfacial widths in polymer blends 41,42,43. Recently, Aarts et al. have even succeeded in visualizing capillary waves directly in a colloid-polymer mixture 31.

Liquid crystals are a particularly interesting class of soft materials, because of the additional aspect of orientational order. The present study is partly motivated by a recent simulation studies of the nematic/isotropic interface in a system of ellipsoids 37, where it was found that (i) the capillary wave spectrum is anisotropic, and (ii) the interface is rougher on short length scales than one would expect from Eq. (1). While the second observation is not unusual 26 and has been predicted theoretically for systems with short range [24] and long range interactions [25], the first is clearly characteristic for liquid crystal interfaces. In Sec. 3 we will analyze it within the Landau-de Gennes theory. In particular, we will discuss the influence of elastic interactions. We find that the anisotropy of the spectrum can already been explained within an approximation that excludes elastic interactions. However, adding the latter changes the spectrum qualitatively to the effect that the leading surface tension term becomes isotropic, and the anisotropy is governed by additional higher order terms. We summarize and conclude in Sec. 4 .

\section{Berreman anchoring on rough and patterned surfaces}

We consider a nematic liquid crystal confined by a surface at $z=h(x, y)$, which locally favors planar anchoring (i.e., alignment parallel to the surface). The surface fluctuations $h(x, y)$ are assumed to be small. The bulk free energy is given by the Frank elastic energy 1,44

$$
\begin{aligned}
F_{F}= & \frac{1}{2} \int d x d y \int_{-\infty}^{h(x, y)} d z\left\{K_{1}(\nabla \mathbf{n})^{2}+K_{2}(\mathbf{n}(\nabla \times \mathbf{n}))^{2}\right. \\
& \left.+K_{3}(\mathbf{n} \times(\nabla \times \mathbf{n}))^{2}\right\}
\end{aligned}
$$

where $\mathbf{n}$ is the director, a vector of length unity which describes the local orientation of the liquid crystal, and $K_{i}$ are the elastic constants (splay, twist and bend). Since the surface favors planar alignment and the bulk fluctuations are small, we assume that the orientation of the director deep in the bulk, $\mathbf{n}_{b}$, lies in the $(x, y)$-plane and that local deviations from $\mathbf{n}_{b}$ are small. Without loss of generality, we take $\mathbf{n}_{b}$ to point in the $y$ direction. Hence we rewrite the director as

$$
\mathbf{n}=\left(u, \sqrt{1-u^{2}-v^{2}}, v\right)
$$

and expand the free energy (2) up to second order in powers of $u, v$, and $h$. This gives

$$
\begin{aligned}
F_{F} \approx & \frac{1}{2} \int d x d y \int_{-\infty}^{0} d z\left\{K_{1}\left(\partial_{x} u+\partial_{z} v\right)^{2}\right. \\
& \left.+K_{2}\left(\partial_{z} u-\partial_{x} v\right)^{2}+K_{3}\left(\left(\partial_{y} u\right)^{2}+\left(\partial_{y} v\right)^{2}\right)\right\}
\end{aligned}
$$

Next we perform a two dimensional Fourier transform $(x, y) \rightarrow \mathbf{q}$. Minimizing $F_{F}$ in the bulk leads to the EulerLagrange equations

$$
\left(\begin{array}{cc}
K_{2} \partial_{z z}-K_{1} q_{x}^{2}-K_{3} q_{y}^{2} & -i q_{x}\left(K_{2}-K_{1}\right) \partial_{z} \\
-i q_{x}\left(K_{2}-K_{1}\right) \partial_{z} & K_{1} \partial_{z z}-K_{2} q_{x}^{2}-K_{3} q_{y}^{2}
\end{array}\right)\left(\begin{array}{c}
u \\
v
\end{array}\right)=0 .
$$

For the boundary conditions $(u, v) \rightarrow 0$ for $z \rightarrow-\infty$ and $(u, v)=\left(u_{0}, v_{0}\right)$ at $z=0$, the solution has the form

$$
\left(\begin{array}{l}
u \\
v
\end{array}\right)=\left(\begin{array}{cc}
i q_{x} & -\lambda_{2} \\
\lambda_{1} & i q_{x}
\end{array}\right)\left(\begin{array}{c}
c_{1} \exp \left(\lambda_{1} z\right) \\
c_{2} \exp \left(\lambda_{2} z\right)
\end{array}\right)
$$

with the coefficients

$$
\left(\begin{array}{l}
c_{1} \\
c_{2}
\end{array}\right)=\frac{1}{\lambda_{1} \lambda_{2}-q_{x}^{2}}\left(\begin{array}{cc}
i q_{x} & \lambda_{2} \\
-\lambda_{1} & i q_{x}
\end{array}\right)\left(\begin{array}{l}
u_{0} \\
v_{0}
\end{array}\right)
$$

and the inverse decay lengths

$$
\lambda_{1,2}^{2}=q_{x}^{2}+q_{y}^{2} \frac{K_{3}}{K_{1,2}} .
$$

Inserting that into the Frank energy (4), one obtains

$$
F_{F}=\frac{1}{2} \int d \mathbf{q} \frac{q_{y}^{2} K_{3}}{\lambda_{1} \lambda_{2}-q_{x}^{2}}\left\{\lambda_{1}\left|u_{0}\right|^{2}+\lambda_{2}\left|v_{0}\right|^{2}+2 q_{x} \Im\left(v_{0}^{*} u_{0}\right)\right\},
$$

where $u_{0}(\mathbf{q})$ and $v_{0}(\mathbf{q})$ are the values of $u(\mathbf{q}), v(\mathbf{q})$ at the surface. This is a general result, which we shall also use in Sec. 3

Now we study more specifically a liquid crystal in contact with a fixed patterned surface (fixed $h(x, y)$ ), which 
anchors in an unspecifically planar way. The surface energy is taken to be of Rapini Papoular type [45]

$$
F_{s}=\sigma_{0} \int d A\left(1+\frac{\alpha_{0}}{2}\left(\mathbf{n}_{0} \mathbf{N}\right)^{2}\right) \quad \text { with } \quad \sigma_{0}>0,
$$

where $d A=d x d y \sqrt{1+\left(\partial_{x} h\right)^{2}+\left(\partial_{y} h\right)^{2}}$ is the local surface area element at $(x, y)$, and

$$
\mathbf{N}=\frac{1}{\sqrt{1+\left(\partial_{x} h\right)^{2}+\left(\partial_{y} h\right)^{2}}}\left(-\partial_{x} h,-\partial_{y} h, 1\right)
$$

the local surface normal. Planar anchoring implies $\alpha_{0}>$ 0 . As before, we rewrite the director at the surface $\mathbf{n}_{0}$ in terms of local deviations $u_{0}, v_{0}$, according to Eq. (31), perform a Fourier transform $(x, y) \rightarrow \mathbf{q}$, and expand $F_{s}$ up to second order in $u_{0}, v_{0}$, and $h$. Omitting the constant contribution $\sigma_{0} A$, this gives

$$
F_{s}=\frac{\sigma_{0}}{2} \int d \mathbf{q}\left\{|h|^{2} q^{2}+\alpha_{0}\left|v_{0}-i q_{y} h\right|^{2}\right\} .
$$

We combine (8) and (11) and minimize the total free energy $F=F_{F}+F_{s}$ with respect to $u_{0}$ and $v_{0}$. The result is

$$
F=\frac{1}{2} \int d \mathbf{q}|h|^{2} q^{2}\left\{\sigma_{0}+K_{3} q \frac{\hat{q}_{y}^{4} \hat{\kappa}\left(\hat{q}_{y}^{2}\right)}{1+q \hat{q}_{y}^{2} \hat{\kappa}\left(\hat{q}_{y}^{2}\right) K_{3} / \sigma_{0} \alpha_{0}}\right\}
$$

with $\hat{q}_{y}=q_{y} / q$ and

$$
\hat{\kappa}\left(\hat{q}_{y}^{2}\right)=1 / \sqrt{1+\hat{q}_{y}^{2}\left(K_{3} / K_{1}-1\right)} .
$$

The result can be generalized easily for the case that the bulk director $\mathbf{n}_{b}$ points in an arbitrary planar direction

$$
\mathbf{n}_{b}=\left(\cos \phi_{0}, \sin \phi_{0}, 0\right)
$$

by simply replacing $\hat{q}_{y}$ with $\mathbf{n}_{b} \mathbf{q} / q$.

Eq. (12) already shows that the bulk director will favor orientations where the amplitudes $|h(\mathbf{q})|$ are small, i.e., the roughness is low. To quantify this further, we expand the integrand of (12) for small wave vectors in powers of $q$. The angle dependent part of the free energy as a function of the bulk director angle $\phi_{0}$ then takes the form

$$
F\left(\phi_{0}\right)=\frac{K_{3}}{2} \int_{-\pi}^{\pi} d \phi \cos ^{4}\left(\phi-\phi_{0}\right) \hat{\kappa}\left(\cos ^{2}\left(\phi-\phi_{0}\right)\right) H(\phi),
$$

where the roughness spectrum $|h(\mathbf{q})|^{2}$ enters the anchoring energy solely through the function

$$
H(\phi)=\int_{0}^{\infty} d q q^{4}|h(\mathbf{q})|^{2} .
$$

It is convenient to expand $H(\phi)$ into a Fourier series with coefficients

$$
H_{n}=\frac{1}{2 \pi} \int d \phi H(\phi) e^{-i n \phi}=:-\left|H_{n}\right| e^{i n \alpha_{n}} .
$$

Similarly, we define

$$
c_{n}=\frac{1}{2 \pi} \int d \phi \cos ^{4} \phi \hat{\kappa}\left(\cos ^{2} \phi\right) e^{-i n \phi} .
$$

The coefficients $c_{n}$ are real and vanish for odd $n$. In the case $K_{3}=K_{1}$ (e.g., in the Landau-de Gennes theory, Sec. 3.1), one has $\hat{\kappa} \equiv 1$, and the series $c_{n}$ stops at $|n|=4$ with $c_{2}=1 / 4$ and $c_{4}=1 / 16$. In real materials [1, the elastic constant $K_{3}$ is typically larger than $K_{1}$ by a factor of $1-3$, and the series does not stop. However, the coefficients for $|n| \leq 4$ remain positive, and the coefficients for $|n|>4$ become very small, such that they may be neglected.

Omitting constant terms that do not depend on $\phi_{0}$, the anchoring energy can then be written as

$$
F\left(\phi_{0}\right)=-\pi K_{3} \sum_{n=2,4} c_{n}\left|H_{n}\right| \cos n\left(\phi_{0}-\alpha_{n}\right) .
$$

The anchoring angle is the angle that minimizes $F\left(\phi_{0}\right)$. In general, the $n=2$ term will dominate, and one gets approximately

$$
\bar{\phi}_{0} \approx \alpha_{2}=\frac{1}{2} \arg \left(-\frac{1}{2 \pi} \int d \phi e^{-2 i \phi} H(\phi)\right) .
$$

We note that the angles $\alpha_{n}$ in Eq. (17) correspond to directions where the height fluctuations are small, because the contributions of $H_{0}$ and $H_{n}$ to the spectral function $H(\phi)$ have opposite signs. Hence Eq. (20) implies that the surface aligns the nematic fluid in a direction where the surface is smooth. At given anchoring angle $\bar{\phi}_{0}$, we can also calculate the anchoring strength. To this end, we expand the anchoring energy about $\bar{\phi}_{0}$ and obtain $F\left(\phi_{0}\right)=F\left(\bar{\phi}_{0}\right)+\frac{W}{2}\left(\phi_{0}-\bar{\Phi}_{0}\right)^{2}$ with the anchoring strength

$$
W=\pi K_{3} \sum_{n=2,4} n^{2} c_{n}\left|H_{n}\right| \cos n\left(\bar{\phi}_{0}-\alpha_{n}\right)
$$

We conclude that elastic interactions in nematic liquid crystals on anisotropically rough surfaces induce an anchoring energy in a direction of low roughness. The central quantities characterizing the surface roughness are the two coefficients $H_{2,4}$ defined by Eqs. (16) and (17). These quantities determine the anchoring strength (Eq. (21), and the anchoring angle (Eq. (20)). The anchoring mechanism only requires an unspecific tendency of the liquid crystal to align parallel to the interface (Eq. (9)). Given such a tendency, the anchoring energy no longer depends on the surface parameters, $\alpha_{0}$ and $\sigma_{0}$. The only relevant material parameters are the splay and bend elastic constants in the bulk, $K_{1}$ and $K_{3}$, and the squared surface anisotropy, which is characterized by the coefficients $H_{2,4}$.

We note that our treatment premises that the nematic liquid stays perfectly ordered at the surface. In reality, rough surfaces may reduce the order, which in turn influences the anchoring properties [46,47. This has not been considered here. 


\section{Capillary waves at the nematic/isotropic interface}

In this section we study the capillary wave spectrum of freely undulating nematic/isotropic (NI) interfaces. The problem is similar to that considered in the previous section (Sec. 2), with two differences: (i) The interface position $h(x, y)$ is free and subject to thermal fluctuations, and (ii) the nematic order at the interface drops smoothly to zero. The second point implies, among other, that the elastic constants are reduced in the vicinity of the interface.

In many systems, the anchoring at NI-interfaces is planar. As a zeroth order approach, we neglect the softness of the profile and approximate the interfacial structure by a steplike structure (sharp-kink approximation), and the interfacial free energy by Eq. (12) with effective parameters $\sigma_{0}$ and $\alpha_{0}$. Generally the capillary waves of an interface with an interfacial free energy of the form

$$
F=\frac{1}{2} \int d \mathbf{q}|h(\mathbf{q})|^{2} \Sigma(\mathbf{q})
$$

are distributed according to

$$
\left.\left\langle|h(\mathbf{q})|^{2}\right\rangle=k_{B} T / \Sigma(\mathbf{q})\right) .
$$

Thus the free energy (12) yields the capillary wave spectrum

$$
\frac{k_{B} T / \sigma_{0}}{\left\langle|h(\mathbf{q})|^{2}\right\rangle} \approx q^{2}+q^{3} \frac{K_{3}}{\sigma_{0}} \hat{q}_{y}^{4} \hat{\kappa}-q^{4} \frac{K_{3}^{2} \hat{q}_{y}^{6} \hat{\kappa}^{2}}{\sigma_{0}^{2} \alpha_{0}}+\cdots
$$

As before, $\hat{q}_{y}$ is the component of the unit vector $\mathbf{q} / q$ in the direction of the bulk director.

The result (24) shows already the three remarkable features, which will turn out to be characteristic for the NI interface. First, the capillary wave spectrum is anisotropic, the capillary waves in the direction parallel to the director $\left(\hat{q}_{y}\right)$ being smaller than in the direction perpendicular to the director. Second, the leading (quadratic) term is still isotropic; the anisotropy enters through the higher order terms. Third, in contrast to simple fluids with short range interactions, the capillary wave spectrum cannot be expanded in even powers of $q$, but it contains additional cubic (and higher order odd) terms. This implies that the capillary wave spectrum is nonanalytic in the limit $\mathbf{q} \rightarrow 0$.

These findings are gratifying. However, the sharp kink description of the NI interface is inadequate. The Frank free energy (2) describes nematic liquid crystals with constant local order parameter, whereas at NI interfaces, the nematic order parameter drops softly to zero. Moreover, the surface anchoring at NI interfaces is an intrinsic property of the interface, which depends itself on the local elastic constants. We will now consider our problem within a unified theory for nematic and isotropic liquid crystals, the Landau-de Gennes theory.

\subsection{Landau-de Gennes theory}

The Landau-de Gennes theory is based on a free energy expansion in powers of a symmetric and traceless $(3 \times 3)$ order tensor field $\mathbf{Q}(\mathbf{r})$.

$$
\begin{aligned}
F=\int d \mathbf{r} & \left\{\frac{A}{2} \operatorname{Tr}\left(\mathbf{Q}^{2}\right)+\frac{B}{3} \operatorname{Tr}\left(\mathbf{Q}^{3}\right)+\frac{C_{1}}{4} \operatorname{Tr}\left(\mathbf{Q}^{2}\right)^{2}+\frac{C_{2}}{4} \operatorname{Tr}\left(\mathbf{Q}^{4}\right)\right. \\
& \left.+\frac{L_{1}}{2} \partial_{i} Q_{j k} \partial_{i} Q_{j k}+\frac{L_{2}}{2} \partial_{i} Q_{i j} \partial_{k} Q_{k j}\right\}
\end{aligned}
$$

Following a common assumption, we neglect the possibility of biaxiality and rewrite the order tensor as 48]

$$
Q_{i j}(\mathbf{r})=\frac{1}{2} S(\mathbf{r})\left(3 n_{i}(\mathbf{r}) n_{j}(\mathbf{r})-\delta_{i j}\right)
$$

Here $S$ is the local order parameter, and $\mathbf{n}$ a unit vector characterizing the local director. In the homogeneous case $\left(\partial_{i} Q_{j k} \equiv 0\right)$, the free energy (25) predicts a first order transition between an isotropic phase (I) with $S=$ 0 and an oriented nematic phase (N) with $S=S_{0}=$ $-2 / 9 B /\left(C_{1}+C_{2} / 2\right)$. We recall briefly the properties of a flat NI interface at coexistence for a system with fixed director $\mathbf{n}$, as obtained from minimizing (25) 48: The order parameter profile has a simple tanh form

$$
S(z)=S_{0} \bar{S}(z / \xi) \quad \text { with } \quad \bar{S}(\tau)=1 /\left(e^{\tau}+1\right) .
$$

The interfacial width

$$
\xi=\xi_{0} \sqrt{1+\alpha(\mathbf{n N})^{2}} \quad\left(\xi_{0}=\frac{2}{S_{0}} \sqrt{\frac{L_{1}+L_{2} / 6}{3\left(C_{1}+C_{2} / 2\right)}}\right)
$$

and the interfacial tension

$$
\sigma=\sigma_{0} \sqrt{1+\alpha(\mathbf{n N})^{2}} \quad\left(\sigma_{0}=\frac{3\left(C_{1}+C_{2} / 2\right)}{16} S_{0}^{4} \xi_{0}\right)
$$

both depend in the same way on the angle between the director $\mathbf{n}$ and the surface normal $\mathbf{N}$, via the parameter

$$
\alpha=\frac{1}{2} \frac{L_{2}}{L_{1}+L_{2} / 6} .
$$

The quantity $\sigma_{0}$ sets the energy scale, $\xi_{0}$ the length scale, and $S_{0}$ the "order parameter scale". (Note that $S$ can be rescaled even though it is dimensionless). Hence only one characteristic material parameter remains, e.g., the parameter $\alpha$. In the following, we shall always use rescaled quantities $S \rightarrow S / S_{0}$, length $\rightarrow$ length $/ \xi_{0}$, energy $\rightarrow$ energy $/ \sigma_{0}$. The free energy at coexistence can then be rewritten as 48 .

$$
F=\int d \mathbf{r}\left\{f+g_{1}+g_{2}+g_{3}+g_{4}\right\}
$$

$$
\text { with } \begin{aligned}
f & =3 S^{2}\left(S^{2}-1\right) \quad(\text { at coexistence }) \\
g_{1}= & 3\left((\nabla S)^{2}+\alpha(\mathbf{n} \nabla S)^{2}\right) \\
g_{2}= & 12 \alpha\left((\nabla \mathbf{n})(\mathbf{n} \nabla S)+\frac{1}{2}(\mathbf{n} \times \nabla \times \mathbf{n})(\nabla S)\right) \\
g_{3}= & 3\left((3+2 \alpha)(\nabla \mathbf{n})^{2}+(3-\alpha)(\mathbf{n} \cdot \nabla \times \mathbf{n})^{2}\right. \\
& \left.+(3+2 \alpha)(\mathbf{n} \times \nabla \times \mathbf{n})^{2}\right) .
\end{aligned}
$$


The first term $f(S)$ describes the bulk coexistence, the middle terms $g_{1}$ and $g_{2}$ determine the structure of the interface, and the last term establishes the relation to the Frank elastic energy, Eq. (21). We note that in this version of the Landau-de Gennes theory, the splay and the bend elastic constants are identical, $K_{1}=K_{3}$, hence $\hat{\kappa}\left(\hat{q}_{y}^{2}\right) \equiv 1$ in Eq. (24).

Eq. (31) will be our starting point. As in Sec. 2] we will assume without loss of generality that the interface is on average located at $z=0$, and that the bulk director far from the surface points in the $y$-direction.

\subsection{Constant director approximation}

We return to considering undulating interfaces with varying position $h(x, y)$. In the simplest approach, the director is constant throughout the system, $\mathbf{n} \equiv(0,1,0)$. Elastic interactions are thus disregarded. For the order parameter, we make the Ansatz $S(\mathbf{r})=\bar{S}((z-h(x, y)) / \xi)$, where $\bar{S}$ is the tanh profile from Eq. (27), and the interfacial width $\xi$ varies with the local surface normal $\mathbf{N}$ (10) according to Eq. (28). Inserting this into the free energy (31), and Fourier transforming $(x, y) \rightarrow \mathbf{q}$, one obtains

$$
F=A+\frac{1}{2} \int d \mathbf{q}|h|^{2}\left\{q^{2}+\alpha q_{y}^{2}\right\}
$$

This result is quite robust. As we shall see in Sec. (3.4), it is also obtained with a rather different Ansatz for $S$, as long as the director is kept constant. We can now apply Eq. (23) and obtain the capillary wave spectrum

$$
\frac{k_{B} T / \sigma_{0}}{\left\langle|h(\mathbf{q})|^{2}\right\rangle}=\left(q^{2}+\alpha q_{y}^{2}\right)
$$

(recalling that wave vectors $q$ are given in units of $1 / \xi_{0}$ (28) ), which is anisotropic.

Hence already this simple approach predicts anisotropic capillary wave amplitudes. The capillary waves are weakest in the $y$ direction, which is the direction of the bulk director. It is interesting that we get the anisotropy already at this point. One might have suspected that the dampening of waves parallel to the director is caused by director-director interactions. This turns out not to be the case, instead an interaction between the director and the order parameter gradient $\mathbf{n} \nabla S$ is responsible for the anisotropy. As the director wants to align parallel to the surface, waves parallel to the director have higher energy.

\subsection{Relaxing the director: A variational approach}

Since the interface locally favors parallel anchoring, one would expect that the director follows the undulations of the interface (Fig. 11). This motivates a variational Ansatz $\mathbf{n}=(0, \cos \theta, \sin \theta)$ with

$$
\theta(x, y, z)=g(x, y) \exp \left[\frac{\kappa}{\xi}(z-h(x, y))\right]
$$

As before, we assume that the profile has the form $S=$ $\bar{S}((z-h) / \xi)$. After inserting this Ansatz in (31), expanding in $\theta$ up to second order, and minimizing with respect to $g$, a lengthy calculation yields the surface free energy (omitting constant terms)

$$
\left.\begin{array}{c}
F=\frac{1}{2} \int d \mathbf{q}|h|^{2}\left\{q^{2}+\alpha q_{y}^{2}\left(1-\frac{3 c^{2}}{c_{1}+c_{2} q_{y}^{2}+c_{3} q_{x}^{2}}\right)\right\}(35) \\
\text { with } \quad c=\left[\left(\begin{array}{c}
3 \\
1+\kappa
\end{array}\right)_{\Gamma}^{-1}-2 \kappa\left(\begin{array}{l}
2 \\
\kappa
\end{array}\right)_{\Gamma}^{-1}\right] \\
c_{1}=\left[\left(\begin{array}{c}
3 \\
1+2 \kappa
\end{array}\right)_{\Gamma}^{-1}-2 \kappa\left(\begin{array}{c}
2 \\
2 \kappa
\end{array}\right)_{\Gamma}^{-1}+\kappa^{2} \frac{3+\alpha}{\alpha}\left(\begin{array}{c}
1 \\
\kappa-1
\end{array}\right)_{\Gamma}^{-1}\right.
\end{array}\right]
$$

where we have defined generalized binomial coefficients,

$$
\left(\begin{array}{l}
n \\
a
\end{array}\right)_{\Gamma}=\frac{\Gamma(n+1)}{\Gamma(a+1) \Gamma(n-a+1)} .
$$

As a consistency check, we also inspected the result for $\theta(x, y, z)$ directly. It is proportional to $\partial_{y} h$ as expected.

The comparison of the free energy (35) with the corresponding result for fixed director, Eq. (32), shows that the anisotropy of the surface fluctuations is reduced. For a further analysis, it would be necessary to minimize the free energy expression (35) with respect to the variational parameter $\kappa$. Unfortunately, $\kappa$ enters in such a complicated way, that this turns out to be unfeasible. Numerically, we find that the capillary wave spectrum, obtained via Eq. (23), varies only little with $\kappa$. For any reasonable value of $\kappa$, i.e., $\kappa^{-1}>2$, the result differs from that obtained with the constant director approximation (Eq. (33)) by less than one percent. Within the present approximation, the effect of relaxing the director is negligeable. This is mostly due to the fact that Eq. (34) still imposes rather rigid constraints on the director variations in the nematic fluid.

\subsection{Local profile approximation}

A more general solution can be obtained with the additional approximation that the width of the interface is small, compared to the relevant length scales of the interfacial undulations. In that case, the interface and the bulk can be considered separately, and we can derive analytical expressions for the capillary wave spectrum. The assumption that length scales can be separated is highly questionable, because interfacial undulations are present on all length scales down to the molecular size. Nevertheless, computer simulations of other systems (Ising models 40] and polymer blends 36 ) have shown that intrinsic profile models can often describe the structure of interfaces quite successfully.

We separate the free energy (31) into an interface and bulk contribution, $F=F_{S}+F_{F}$. The bulk contribution 


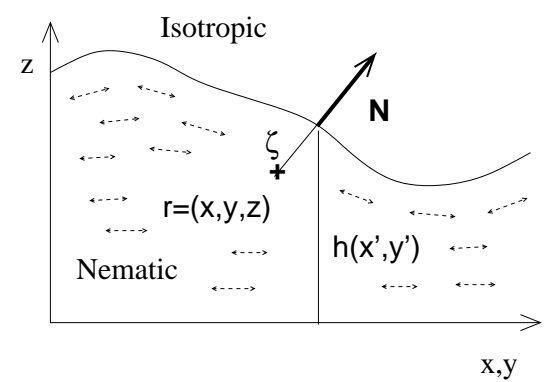

Fig. 1. Nematic-Isotropic interface with local coordinates.

$F_{F}$ has the form (2) with the elastic constants $K_{1}=K_{3}=$ $6(3+2 \alpha)$ and $K_{2}=6(3-\alpha)$, and accounts for the elastic energy within the nematic region. The integrand in the expression for the remaining surface free energy $F_{S}$ vanishes far from the surface. We assume that the local order parameter profile has mean-field shape in the direction perpendicular to the surface. More precisely, we make the Ansatz

$$
S(\mathbf{r})=\bar{S}(\zeta / \xi), \quad \nabla S \approx \frac{1}{\xi}\left(\left.\frac{d \bar{S}}{d \tau}\right|_{\tau=\zeta / \xi} \mathbf{N}\right.
$$

(cf. (27), (28), where $\mathbf{N}$ is the local surface normal as usual, and $\zeta$ is the distance between $\mathbf{r}$ and the closest interface point. The $(x, y)$ coordinates at this point are denoted $\left(x^{\prime}, y^{\prime}\right)$. (see Fig. 1). To evaluate $F_{S}$, we make a coordinate transformation $\mathbf{r} \rightarrow\left(x^{\prime}, y^{\prime}, \zeta\right)$ and integrate over $\zeta$. The relation between the coordinates is $\mathbf{r}=\left(x^{\prime}, y^{\prime}, h\left(x^{\prime}, y^{\prime}\right)\right)+$ $\mathbf{N} \zeta$, and the Jacobi determinant for the integral is in second order of $h$

$1+\frac{1}{2}\left(\left(\partial_{x} h\right)^{2}+\left(\partial_{y} h\right)^{2}\right)-\zeta\left(\partial_{x x} h+\partial_{y y} h\right)+\zeta^{2}\left(h_{x x} h_{y y}-h_{x y} h_{y x}\right)$.

We begin with reconsidering the constant director case, $\mathbf{n}=$ const. The Frank free energy then vanishes, and the surface free energy takes exactly the form of Eq. (32). Hence the present approximation leads to the same expression as the approximation taken in Sec. [3.2. This underlines the robustness of the result (33).

In the general case, we must make an Ansatz for the variation of the director $\mathbf{n}$ in the vicinity of the surface. We assume that it varies sufficiently slowly, so that we can make a linear approximation

$$
\mathbf{n}\left(x^{\prime}, y^{\prime}, \zeta\right) \approx \mathbf{n}\left(x^{\prime}, y^{\prime}, 0\right)+\zeta(\mathbf{N} \cdot \nabla) \mathbf{n}
$$

As before, we take the bulk director to point in the $y$ direction. The local director deviations in the $x$ and $z$ direction are parametrized in terms of two parameters $u$ and $v$ according to Eq. (3).

After inserting Eqs. (36) and (37) into Eq. (31), expanding up to second order in $h, u$, and $v$, and some partial integrations, we obtain the surface free energy

$$
F_{S}=\int d x d y\left(g_{1 s}+g_{2 s}+g_{3 s}\right)
$$

with

$$
\begin{aligned}
g_{1 s}=1 & +\frac{1}{2}\left(\left(\partial_{x} h\right)^{2}+\left(\partial_{y} h\right)^{2}\right) \\
& +\frac{\alpha}{2}\left(\partial_{y} h-v_{0}\right)^{2}+\frac{\pi^{2}-6}{6} \alpha\left(\partial_{z} v_{0}\right)^{2} \\
g_{2 s}= & 3 \alpha\left(2\left(\partial_{y} h\right)\left(\partial_{x} u_{0}+\partial_{z} v_{0}\right)+\left(\partial_{x x} h+\partial_{y y} h\right)\left(\partial_{y} v_{0}\right)\right. \\
& -\left(\partial_{x} h\right)\left(\partial_{y} u_{0}\right)-v_{0}\left(\partial_{z} u_{0}-3 \partial_{x} u_{0}\right) \\
+ & \left.2\left(\partial_{z} v_{0}\right)\left(\partial_{x} u_{0}+\partial_{z} v_{0}\right)+2\left(\partial_{z} u_{0}\right)\left(\partial_{z} u_{0}-\partial_{x} v_{0}\right)\right) \\
g_{3 s}=3( & (3+2 \alpha)\left(\partial_{x} u_{0}+\partial_{z} v_{0}\right)^{2}+(3-\alpha)\left(\partial_{z} u_{0}-\partial_{x} v_{0}\right)^{2} \\
& \left.+(3+2 \alpha)\left(\left(\partial_{y} u_{0}\right)^{2}+\left(\partial_{y} v_{0}\right)^{2}\right)\right),
\end{aligned}
$$

where $u_{0}$ and $v_{0}$ are the values of $u$ and $v$ at the interface. The first contribution $g_{1 s}$ describes the effect of the anisotropic local surface tension. The second contribution $g_{2 s}$ arises from the coupling between the director variations $\partial_{i} n$ with the order parameter variation $\nabla S$ at the interface. The last contribution $g_{3 s}$ accounts for the reduction of the Frank free energy in the interface region.

The ensuing procedure is similar to that of Sec. 2 We first minimize the bulk free energy $F_{F}$, which leads in the most general case to Eq. (12). Then we minimize the total energy $F=F_{F}+F_{S}$ with respect to $u_{0}$ and $v_{0}$, using Eqs. (5) and (6) to estimate the derivatives $\partial_{z} u_{0}, \partial_{z} v_{0}$. The result has the form (22) and gives the capillary wave spectrum via (23). Unfortunately, the final expression is a rather lengthy, and we cannot give the formula here. We will discuss it further below.

A more concise and qualitatively similar result is obtained with the additional approximation, that the director only varies in the $z$ direction, $u \equiv 0$. In that case, the minimization of the Frank free energy (2) with respect to $v$ yields $v(\mathbf{q}, z)=v_{0} \exp (q \hat{k} z)$ with

$$
\hat{k}\left(\hat{q}_{y}^{2}\right)=\sqrt{\frac{3-\alpha}{3+2 \alpha}} \sqrt{1+\hat{q}_{y}^{2} \frac{3 \alpha}{3-\alpha}}
$$

$\left(\hat{q}_{y}=q_{y} / q\right)$, i.e., at $z=0$ we have $\partial_{z} u_{0}=q \hat{k} u_{0}$. The Frank energy takes the form

$$
F_{F}=\frac{1}{12 \alpha} \int d \mathbf{q}(3+2 \alpha) q \hat{k}\left(\hat{q}_{y}^{2}\right)\left|v_{0}\right|^{2}
$$

This equation replaces Eq. (12). The surface free energy $F_{S}$ (38) is also greatly simplified. After minimizing the sum $F=F_{F}+F_{S}$ with respect to $v_{0}$ and applying Eq. (23), one obtains the capillary wave spectrum

$$
\begin{aligned}
& \frac{k_{B} T / \sigma_{0}}{\left\langle|h(\mathbf{q})|^{2}\right\rangle}=q^{2}+\alpha q_{y}^{2} \\
& \quad \times\left(1-\frac{\left(1-6 q \hat{k}-3 q^{2}\right)^{2}}{1+18 q \hat{k}\left(\left(\frac{1}{3}+\frac{1}{\alpha}\right)(1-2 q \hat{k})+\frac{\pi^{2}-6}{54} q \hat{k}\right)}\right) .
\end{aligned}
$$

Expanding this for small wavevectors $q$ gives

$$
\frac{k_{B} T / \sigma_{0}}{\left\langle|h(\mathbf{q})|^{2}\right\rangle}=q^{2}+\hat{q}_{y}^{2}\left(q^{3} 18(1+\alpha) \hat{k}-q^{4} \cdots\right),
$$




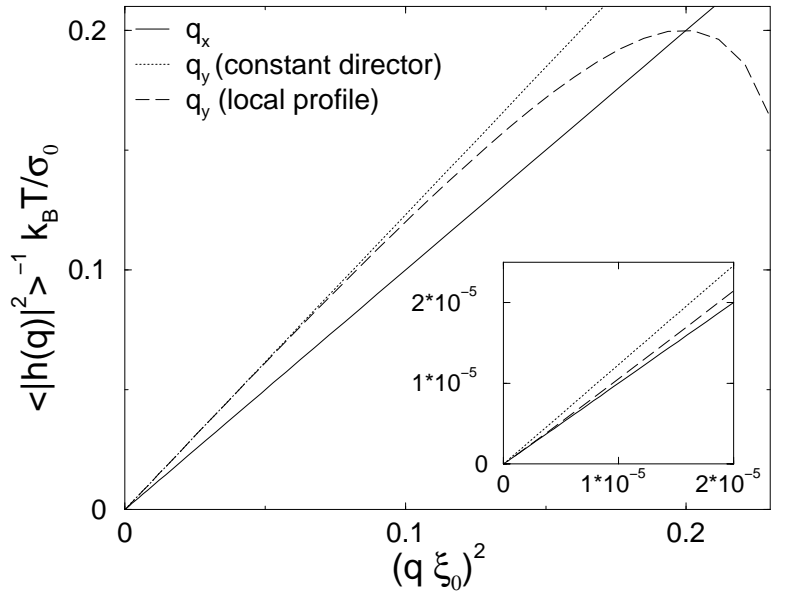

Fig. 2. Capillary wave spectrum in the direction perpendicular to the bulk director $\left(q_{x}\right)$ (solid line) and parallel to the bulk director $\left(q_{y}\right)$ as obtained from the constant director approximation (dotted line) and the local profile approximation (dashed line). In the perpendicular direction, both approaches give the same result. Note the presence of an unphysical pole at $\left(q \xi_{0}\right)^{2}=0.24$. The inset shows a blowup for very small $q$-vectors, illustrating that the spectrum becomes isotropic in the limit $q \rightarrow 0$. The parameter is $\alpha=3 / 13$, corresponding to $L_{1}=2 L_{2}$.

where the coefficient of the fourth order term is negative.

Comparing this solution to Eq. (33), one notes obvious qualitative differences. In the constant director approximation, Eq. (33), one has an anisotropic effective surface tension: The capillary wave spectrum has the form (11) with $\sigma=\sigma_{0}\left(1+\alpha \hat{q}_{y}^{2}\right)$. The present treatment shows that the elastic interactions remove the anisotropy in the surface tension term (order $q^{2}$ ), but introduce new anisotropic terms that are of higher order in $q$. This is consistent with the preliminary results from our earlier zeroth order approach, Eq. (24).

We turn to discussing the full solution of the local profile approximation, where both variations of $u$ and $v$ are allowed. In the directions parallel and perpendicular to the bulk director $(x$ and $y$ ), the capillary wave spectrum turns out to be the same as in (41). It is shown in Fig. 2 for a typical value of $\alpha$ (taken from Ref. 48) and compared to the constant director approximation. The capillary waves in the direction perpendicular to the bulk director (the $x$-direction) are not influenced by the elastic interactions: The amplitudes only contain a $q^{2}$ contribution and are identical in the constant director approximation and the local profile approximation. In contrast, the capillary waves in the direction parallel to the bulk director (the $y$-direction) are to a large extent dominated by the cubic term. The fact that the spectrum becomes isotropic in the limit $q \rightarrow 0$ becomes only apparent at very small $q$ vectors, $q \xi_{0}<0.005$ (see inset of Fig. 2).

The effect of relaxing both $u$ and $v$ in Eq. (3) becomes apparent when examining directions that are intermediate between $x$ and $y$. The expansion of the full solution in powers of $q$ gives an expression that is similar to Eq. (42),

$$
\frac{k_{B} T / \sigma_{0}}{\left\langle|h(\mathbf{q})|^{2}\right\rangle}=q^{2}+\hat{q}_{y}^{2}\left(q^{3} C_{3}\left(\hat{q}_{y}^{2}\right)+q^{4} C_{4}\left(\hat{q}_{y}^{2}\right)+\cdots\right)
$$

but has different coefficients $C_{i}$. Fig. 3 shows the coefficients $C_{3}$ and $C_{4}$ as a function of $\hat{q}_{y}$ and compares them with the corresponding quantities obtained from the simplified solution (41). In the full solution, the $C_{i}$ stay much smaller in the vicinity of $q_{y} \sim 0$. This demonstrates once more that elastic interactions reduce the anisotropy of the capillary waves.

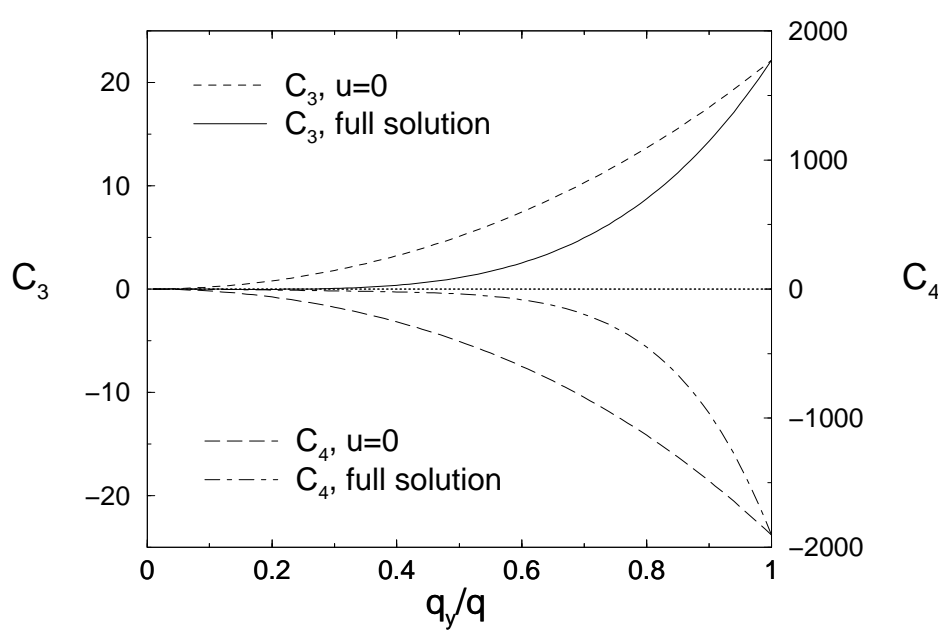

Fig. 3. Coefficients of (a) the cubic term $q^{3}$ and (b) the fourth order term $q^{4}$ vs. $\hat{q}_{y}=q_{y} / q$ in the expansion (43) and (42), at $\alpha=3 / 13$.

Unfortunately, we also find that the free energy $F=$ $F_{F}+F_{S}$ (from Eqs. (12) and (38)), as a functional of $u_{0}(\mathbf{q}), v_{0}(\mathbf{q})$, becomes unstable for larger wavevectors $q$. The instability gives rise to the unphysical pole at $\left(q \xi_{0}\right)^{2}=$ 0.24 in Fig. 22 In the direction $\hat{q}_{y}=1$, the pole is encountered at $q \xi_{0} \sim 0.5$ for all $\alpha$. In other directions, it moves to even smaller $q$ values, which reduces the stability further. At $\alpha=3 / 13$, the free energy is stable only up to $q \xi_{0} \sim 0.023$, corresponding to a length scale of $\sim 270 \xi_{0}$. The approximation is bound to break down on smaller length scales.

Hence the region of validity of the theory is very small. The instability is presumably caused by the way the local profile approximation was implemented. In particular, our estimate for the values of the partial derivatives $\partial_{z} u_{0}$ and $\partial_{z} v_{0}$ at the interface must be questioned. They were obtained from extrapolating the bulk solution, which is however only valid for constant, saturated, order parameter $S \equiv S_{0}$. In order to assess the effect of this constraint, we have considered a second approximation: The derivative $\partial_{z} \mathbf{n}$ at the interface is taken to be independent of the bulk solution. In the interface region, the director is assumed to vary linearly with the slope $\partial_{z} \mathbf{n}$. At the distance $\sim 2.5 \xi_{0}$ from the interface, the profile changes 
continuously to the exponential bulk solution. In this approximation, the value of the derivative $\partial_{z} \mathbf{n}$ at the surface is an additional variable, which can be optimized independently. We have minimized the free energy with this Ansatz and $u \equiv 0$ (i.e., no director variations in the $x$ direction), and calculated the capillary wave spectrum. The result is shown in Fig. 4 The instability from Fig. 2 disappears. The other characteristic features of the spectrum remain. It becomes isotropic for very small wavevectors, $q<0.003 \xi_{0}$, corresponding to length scales of several thousand correlation lengths $\xi_{0}$. On smaller length scales, it is anisotropic. It is worth noting that at least on this level of approximation, the capillary waves in the direction perpendicular to the director (the $x$ direction) are not affected by the elastic interactions.

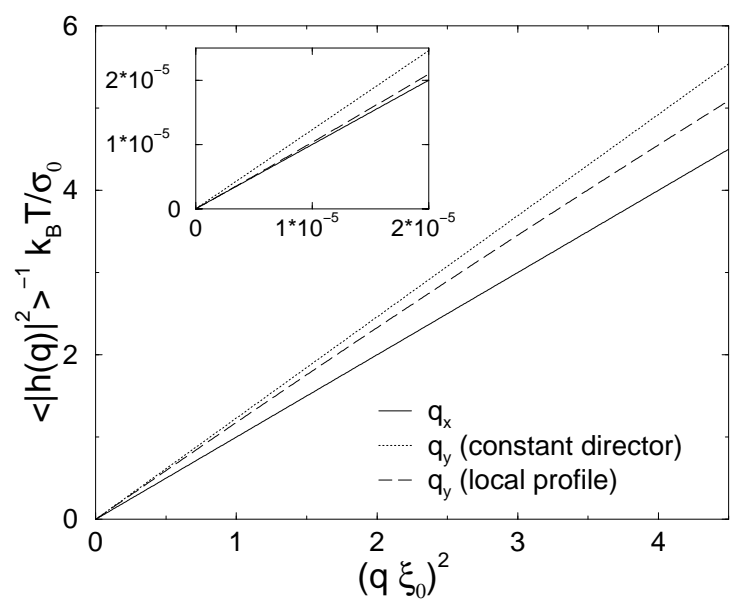

Fig. 4. Capillary wave spectrum from the local profile approximation with independent surface derivative $\partial_{z} u$, compared to the constant director approximation, in the directions parallel and perpendicular to the bulk director. The pole in Fig. 2 has disappeared. At small wavevectors, the curves are the same as in the approximation of Fig. 2

\section{Summary and discussion}

To summarize, we have studied the interplay of elastic interactions and surface undulations for nematic liquid crystals at rough and fluctuating interfaces using appropriate continuum theories: the Frank elastic energy and the Landau-de Gennes theory.

In the first part, we have considered nematic liquid crystals in contact with a surface of given geometry, characterized by a fixed height function $h(x, y)$. We have reanalyzed the effect of Berreman anchoring, i.e., the phenomenon that elastic interactions are sufficient to align liquid crystals exposed to anisotropically rough surfaces. Our treatment allowed to derive explicit equations for the anchoring angle and the anchoring strength for given (arbitrary) height function $h(x, y)$. In particular, we find that the resulting azimuthal anchoring coefficient depends only on the surface anisotropy and the bulk elastic constants $K_{1}$ and $K_{3}$, and not on the chemical surface interaction parameters such as the interfacial tension and the zenithal anchoring strength. The contribution of the surface anisotropy to the anchoring energy has recently been verified by Kumar et al [49. We hope that our results will stimulate systematic experimental research on the role of the elastic constants as well.

In the second part, we have examined the inverse problem, the effect of the nematic order on capillary wave fluctuations of NI interfaces. The work was motivated by a previous simulation study, where it was found that the capillary wave amplitudes were different in the direction parallel and perpendicular to the bulk director. Our analysis shows that this effect can be understood within the Landau-de Gennes theory. As in the simulation, the waves parallel to the director are smaller than those perpendicular. The anisotropy is caused by a coupling term between the director and the order parameter gradient $(\mathbf{n} \nabla S)$, which locally encourages the director to align parallel to the surface, and thus penalizes interfacial undulations in the direction of the director.

The influence of elastic interactions mediated by the nematic bulk fluid was investigated separately. We find that they reduce the anisotropy and change the capillary wave spectrum qualitatively. In the absence of elastic interactions, i.e., with fixed director, the anisotropy manifests itself in an anisotropic surface tension. If one allows for director modulations, the surface tension becomes isotropic, and the anisotropy is incorporated in higher order terms in the wave vector $q$. In particular, we obtain a large anisotropic cubic term. The fourth order term is generally negative, i.e., we have a negative "bending rigidity". This is consistent with previous observations from simulations [37. As we have noted in the introduction, bending rigidities are often negative in fluid-fluid interfaces, for various reasons. In the case of nematic/isotropic interfaces, the elasticity of the adjacent medium provides an additional reason.

We have shown that the higher order terms dominate the capillary wave spectrum on length scales up to several thousand correlation lengths. This has serious practical consequences. The analysis of capillary waves is usually a valuable tool to determine interfacial tensions from computer simulations. In liquid crystals, however, this method must be applied with caution. More generally, our result suggests that the apparent interfacial tension of nematic/isotropic interfaces should be strongly affected by finite size effects. In fact, Vink and Schilling have recently reported that the interfacial tension obtained from computer simulations of soft spherocylinders varies considerably with the system size [50,51].

We thank Marcus Müller for a useful comment, and the German Science Foundation for partial support.

\section{References}

1. P. G. DeGennes and J. Prost, The Physics of Liquid Crystals (Clarendon Press, Oxford, 2nd edn, 1993). 
2. J. M. Geary, J. W. Goodby, A. R. Kmetz, J. S. Patel, J. Appl. Phys. 62, 4100 (1987).

3. J. Cognard, Alignment of Liquid Crystals and their Mixtures (Gordon and Breach, London, 1982).

4. B. Jerome, Rep. Prog. Phys. 54, 391 (1991).

5. E. P. Raynes, U. S. Patent 4,084,884 (1978).

6. P. M. Chaikin, T. C. Lubensky Principles of condensed matter physics. (Cambridge University Press, Cambridge, 1995).

7. M. Papoular, A. Rapini, Sol. State Comm. 7, 1639 (1969).

8. C. Mauguin, B. Soc. Franc. Miner. 34, 71 (1911).

9. M. F. Toney, T. P. Russell, J. A. Logan, H. Kiguchi, J. M. Sands, S. K. Kumar, Nature 374, 709 (1995).

10. S. W. Lee, J. Yoon, H. C. Kim, B. Lee, T. Y. Chang, M. Ree Macromolecules 36, 9905 (2003).

11. B. W. Lee, N. A. Clark, Science 291, 2576 (2001).

12. B. W. Lee, D. Sagong, Mol. Crys. Liqu. Crys. 364, 519 (2001).

13. A. Rastegar, M. Skarabot, B. Blij, T. Rasing J. Appl. Phys. 89, 960 (2001).

14. M. Behdani, S. H. Keshmiri, S. Soria, M. A. Bader, J. Ihlemann, G. Marowsky, T. Rasing, Appl. Phys. Lett. 82, 2553 (2003).

15. D. W. Berreman, Phys. Rev. Lett. 28, 1683 (1972).

16. S. Faetti, Phys. Rev. A 36, 408 (1987).

17. J. B. Fournier, P. Galatola, Phys. Rev. E 60, 2404 (1999).

18. J. B. Fournier, P. Galatola, Eur. Phys. J. E 2, 59 (2000).

19. M. V. von Smoluchowski, Ann. Phys. 25, 205 (1908).

20. F. P. Buff, R. A. Lovett, F. H. Stillinger, Phys. Rev. Lett. 15, 621 (1965).

21. J. S. Rowlinson and B. Widom, Molecular Theory of Capillarity (Clarendon, Oxford, 1982).

22. J. D. Weeks, J. Chem. Phys. 67, 3106 (1977).

23. D. Bedeaux and J. D. Weeks, J. Chem. Phys. 82, 972 (1985).

24. A. O. Parry, C. J. Boulter, J. Phys.: Cond. Matt. 6, 7199 (1994).

25. K. R. Mecke, S. Dietrich, Phys. Rev. E 59, 6766 (1999).

26. J. Stecki, J. Chem. Phys. 114, 7574 (2001).

27. A. K. Doerr, M. Tolan, W. Prange, J.-P. Schlomka, T. Seydel, W. Press, Phys. Rev. Lett. 83, 3470 (1999).

28. C. Fradin, A. Braslau, D. Luzet, D. Smilgies, M. Alba, N. Boudet, K. Mecke, J. Daillant, Nature 403, 871 (2000).

29. S. Mora, J. Daillant, K. Mecke, D. Luzet, A. Braslau, M. Alba, B. Struth, Phys. Rev. Lett. 90, 216101 (2003).

30. D. Li, B. Yang, B. Lin, M. Meron, J. Gebhardt, T. Graber, S. A. Rice, Phys. Rev. Lett. 92, 136102 (2004).

31. D. G. A. L. Aarts, M. Schmidt, H. N. W. Lekkerkerker, Science 304, 847 (2004).

32. K. K. Mon, D. P. Landau, S. Stauffer, Phys. Rev. B 42, 545 (1990).

33. F. Schmid, K. Binder, Phys. Rev. B 46, 13553 (1992).

34. A. Werner, F. Schmid, M. Müller, K. Binder, J. Chem. Phys. 107, 8175 (1997).

35. M. D. Lacasse, G. S. Grest, A.J. Levine, Phys. Rev. Lett. 80, 309(1998).

36. A. Werner, F. Schmid, M. Müller, K. Binder, Phys. Rev. E 59, 728 (1999).

37. N. Akino, F. Schmid, M. P. Allen, Phys. Rev. E 63, 041706 (2001).

38. R. L. C. Vink, J. Horbach, K. Binder, J. Chem. Phys. 122, 134905 (2005).

39. G. Germano, F. Schmid, submitted to J. Chem. Phys. (2005).
40. M. Müller, G. Münster, J. Stat. Phys. 118, 669 (2005).

41. M. Sferrazza, C. Xiao, R. A. L. Jones, D. G. Bucknall, J. Webster, J. Penfold, Phys. Rev. Lett. 78, 3693 (1997).

42. T. Kerle, J. Klein, K. Binder, Eur. Phys. J. B 7, 401 (1999).

43. C. Carelli, R. A. L. Jones, R. N. Young, R. Cubitt, R. Krastev, T. Gutberleg, R. Dalgliesh, F. Schmid, M. Sferrazza, Europhys. Lett. E 71, 763 (2005).

44. F. C. Frank, Discuss. Farady Soc. 25, 19 (1958).

45. A. Rapini, M. Papoular, J. Phys. (France) Coll. 30, C4 (1969).

46. J. Papanek, Ph. Martinot-Lagarde, J. Phys. II France 6, 205 (1996).

47. D. L. Cheung, F. Schmid, J. Chem. Phys. 122, 074902 (2005).

48. P. Sheng and E. B. Priestley, in Introduction to Liquid Crystals, chap. 10, pp. 143, E. B. Priestley, P. J. Wojtowicz., and P. Sheng P. eds. (Plenum Press, New York, 1975) .

49. S. Kumar, J.-H. Kim, Y. Shi, Phys. Rev. Lett. 94, -77803 (2005).

50. R. L. C. Vink, S. Wolfsheimer, T. Schilling, J. Chem. Phys. 123, 074901 (2005).

51. R. L. C. Vink, T. Schilling, Phys. Rev. E 71, 051716 (2005). 